\title{
Critical role for the chemokine MCP-1/CCR2 in the pathogenesis of bronchiolitis obliterans syndrome
}

\author{
John A. Belperio, ${ }^{1}$ Michael P. Keane, ${ }^{1}$ Marie D. Burdick, ${ }^{1}$ Joseph P. Lynch III, ${ }^{2}$ \\ Ying Ying Xue, ${ }^{1}$ Aaron Berlin, ${ }^{3}$ David J. Ross, ${ }^{1}$ Steven L. Kunkel, ${ }^{3}$ Israel F. Charo, ${ }^{4}$ \\ and Robert M. Strieter ${ }^{1}$
}

\begin{abstract}
${ }^{1}$ Department of Medicine, Division of Pulmonary and Critical Care Medicine, University of California Los Angeles School of Medicine, Los Angeles, California, USA

${ }^{2}$ Department of Internal Medicine, Division of Pulmonary and Critical Care Medicine, and

${ }^{3}$ Department of Pathology, The University of Michigan Medical School, Ann Arbor, Michigan, USA

${ }^{4}$ Gladstone Institute of Cardiovascular Disease, University of California San Francisco, San Francisco, California, USA

Address correspondence to: Robert M. Strieter, Department of Medicine, Division of Pulmonary and Critical Care Medicine, UCLA School of Medicine, Room 14-154 Warren Hall, Box 711922,

900 Veteran Avenue, Los Angeles, California 90024-1922, USA.

Phone: (310) 794-1999; Fax: (310) 794-1998; E-mail: rstrieter@mednet.ucla.edu.
\end{abstract}

Received for publication January 12, 2001, and accepted in revised form June 28, 2001.

Bronchiolitis obliterans syndrome (BOS) is the major limitation to survival after lung transplantation. Acute rejection, its main risk factor, is characterized by perivascular/bronchiolar leukocyte infiltration. BOS is characterized by persistent peribronchiolar leukocyte recruitment leading to airway fibrosis and obliteration. The specific mechanism(s) by which these leukocytes are recruited are unknown. Because MCP-1, acting through its receptor CCR2, is a potent mononuclear cell chemoattractant, we hypothesized that expression of this chemokine during an allogeneic-response promotes persistent recruitment of leukocytes and, ultimately, rejection. We found that elevated levels of biologically active MCP-1 in human bronchial lavage fluid (BALF) were associated with the continuum from acute to chronic allograft rejection. Translational studies in a murine model of BOS demonstrated increased MCP-1 expression paralleling mononuclear cell recruitment and CCR2 expression. Loss of MCP-1/CCR2 signaling, as seen in CCR2 $2^{-/}$mice or in WT mice treated with neutralizing antibodies to MCP-1, significantly reduced recruitment of mononuclear phagocytes following tracheal transplantation and led to attenuation of BOS. Lymphocyte infiltration was not reduced under these conditions. We suggest that MCP-1/CCR2 signaling plays an important role in recruitment of mononuclear phagocytes, a pivotal event in the pathogenesis of BOS.

J. Clin. Invest. 108:547-556 (2001). DOI:10.1172/JCI200112214.

\section{Introduction}

Lung transplantation is a therapeutic option for end-stage pulmonary disorders. Unfortunately, lung rejection is a common complication with an incidence and severity that exceed those of all other solid organ transplantations (1, 2). Chronic lung allograft rejection, known as bronchiolitis obliterans syndrome (BOS), is the leading cause of mortality after lung transplantation and is the reason the 7year survival after lung transplantation is only $31 \%(1,2)$.

BOS is a chronic inflammatory process, demonstrating features of dysregulated repair. It is characterized by persistent peribronchiolar leukocyte infiltration that eventually disrupts the basement membrane, submucosa, and airway epithelium $(1,2)$. Fibroproliferation follows with increased numbers of mesenchymal cells, submucosal matrix deposition, and granulation tissue formation, ultimately leading to fibro-obliteration of airways $(1,2)$. Importantly, acute lung allograft rejection is the major risk factor for the development of BOS and is characterized by a perivascular/bronchiolar recruitment of mononuclear cells that ultimately leads to architectural destruction $(1,2)$.
BOS is classically described as an ongoing immunological event; however, aggressive immunosuppressive therapy does not affect the naturally occuring development of this disorder $(1,2)$. Moreover, the specific mediators that orchestrate the persistent recruitment of leukocytes leading to fibro-obliteration have not been fully elucidated. MCP- 1 is a CC chemokine, and it is a potent chemoattractant for mononuclear cells, specifically mononuclear phagocytes, $\mathrm{CD}^{2} 5 \mathrm{RO}^{+} \mathrm{T}$ lymphocytes, B cells, and NK cells (3, 4). MCP-1 is involved in chronic inflammatory/fibroproliferative diseases such as rheumatoid arthritis (5). MCP-1 binds and signals through a seven-transmembrane-spanning $G$ protein-coupled receptor, CCR2 (6).

We hypothesize that the continuum of lung allograft rejection from acute to chronic rejection results from a persistent immunological/inflammatory insult to the allograft airways. This persistent insult is due, in part, to the expression of MCP-1 and its interaction with its major mononuclear cell receptor, CCR2, which leads to chronic peribronchiolar leukocyte infiltration and eventual obliteration of the airways. Our studies 
demonstrate that biologically active human MCP-1 in bronchial lavage fluid (BALF) is associated with both acute rejection and BOS. Translational studies in a murine model of BOS using CCR2 $2^{-/-}$compared with $\mathrm{CCR} 2^{+/+}$mice significantly attenuated the pathogenesis of BOS. In addition, neutralizing antibodies to MCP-1 demonstrated a significant reduction in fibroobliteration. These findings may ultimately result in novel therapies designed to modulate MCP-1/CCR2 signaling and retard the development of BOS.

\section{Methods}

Patient population. With Institutional Review Board approval and informed written consent, we prospectively enrolled all patients undergoing both lung and heart-lung transplantation at the University of Michigan Hospital during 1992-1997. Patients were eligible for this study if they survived at least 6 months after transplantation. Of 92 patients who were evaluated, 77 were included in the study (41 female and 36 male; mean age of $51.4 \pm 1.4$ years). Fifteen patients were excluded from the study owing to technical problems in coordinating the collection of the sample.

The transplant recipients were divided into three groups. The first group comprised healthy lung transplant recipients without infection or rejection $(n=30)$, who were undergoing surveillance bronchoscopy. We used their last available BALF in time. The second group comprised lung transplant recipients who developed acute lung allograft rejection $(n=27)$, without concomitant infection as diagnosed by pathological findings on transbronchial biopsy (7). The third group was composed of lung transplant recipients who developed stage II or greater BOS without concomitant infection or acute rejection $(n=20)$. BALFs from patients with infection were excluded as determined by positive microbiology and/or cytology results as described previously (8). The exclusion of any BALF as well as the assignment to the proper group was done prospectively without knowledge of the BALF chemokine levels. All patients were placed on a standard pre- and posttransplantation immunosuppression, bacterial, and viral prophylaxis as described previously (8).

$B A L F$. BALF was obtained from our three groups of lung transplant recipients and processed by methods described previously (8). Cell-free BALF was aliquoted and frozen immediately until thawed for MCP-1 ELISA (8).

Reagents. Polyclonal rabbit anti-human MCP-1, antimurine MCP-1, and goat anti-murine MIP-2-specific anti-serum was produced by the immunization of rabbits or goats with the appropriate recombinant human and murine chemokines (MCP-1 or MIP-2) (R\&D Systems Inc., Minneapolis, Minnesota, USA) in multiple intradermal sites with complete Freund's adjuvant (9-12). The specificity of these antibodies was assessed by Western blot analysis against a panel of other human and murine recombinant cytokines. Antibodies were specific in our sandwich ELISA without cross- reactivity to a panel of cytokines including human and murine IL-1Ra, IL-1, IL-2, IL-6, IL-4, TNF- $\alpha$, IFN- $\gamma$, and members of the CXC and CC chemokine families.

Monocyte chemotaxis. Monocyte chemotaxis was preformed as described previously (8). Briefly, monocytes were prepared from human peripheral blood by FicollHypaque density gradient centrifugation. Five randomly selected patient BALFs from each group were pooled. The samples from each group, $10 \mathrm{ng} / \mathrm{ml}$ of MCP-1, or HBSS was preincubated with either preimmune serum (NRS) or neutralizing rabbit antihuman MCP- 1 antiserum for 30 minutes at $37^{\circ} \mathrm{C}$. Chemotaxis was performed as described previously (8).

Immunohistochemical localization of antigenic human and murine MCP-1. Paraffin-embedded tissue from open lung biopsies from five patients with BOS and five day 7 tracheal allografts and syngeneic controls were processed for immunohistochemical localization of MCP-1, using NRS or polyclonal rabbit anti-human or murine MCP-1 antibodies as described previously (11).

Murine BOS model. We used a well-established and reproducible murine model of BOS involving heterotopic subcutaneous trachea transplantation studies (13-15). The MHC class I-and class II-disparate combination was BALB/c (H-2 $\left.2^{\mathrm{d}}\right)$ to $\mathrm{C} 57 \mathrm{BL} / 6\left(\mathrm{H}-2^{\mathrm{b}}\right)$ (allografts), and $\mathrm{C} 57 \mathrm{BL} / 6\left(\mathrm{H}-2^{\mathrm{b}}\right)$ to $\mathrm{C} 57 \mathrm{BL} / 6\left(\mathrm{H}-2^{\mathrm{b}}\right)$ (syngeneic control).

To assess the inhibition of MCP-1/CCR2 signaling on leukocyte infiltration and fibro-obliteration we performed tracheal allograft experiments with CCR2-/(knockout mice) versus CCR2 ${ }^{+/+}$(wild-type) recipient mice. A breeding colony containing both murine genotypes were maintained under specific pathogen-free conditions as described previously $(16,17)$. CCR2 ${ }^{+/+}$and $\mathrm{CCR}^{-/-}$mice were generated from mating homozygous mice of the same genetic background (C57BL/6 $\times$ $129 \mathrm{~Sv})$ and were intercrossed for five to seven generations to establish the C57BL/ 6 strain $(16,17)$.

We evaluated differences in the immune response from CCR2 $2^{-/-}$versus $\mathrm{CCR} 2^{+/+}$mice by performing transplants with CCR2 $2^{-/-}$versus $\mathrm{CCR} 2^{+/+}$tracheas into BALB/c. In addition, allografts (BALB/c trachea to $\mathrm{C} 57 \mathrm{BL} / 6)$ were passively immunized with intraperitoneal injections of $0.5 \mathrm{ml}$ of anti-murine MCP-1 serum or NRS on days 1 , $3,5,7,9,11$, and 13 after transplantation to further evaluate the role of MCP-1/CCR2 signaling during BOS.

Histopathological grading of BOS. Six random paraffinembedded tissue sections for six different trachea allografts were stained with Masson's trichrome and hematoxylin and eosin (H\&E) at days 3, 7, 14, and 21. The histopathology was blindly reviewed with a modified histological scoring system as described previously (13, 18). All qualitative histological changes were noted, and four easily identifiable pathological processes were scored on a scale of $0-4(0=$ normal, $1=$ mild, $2=$ moderate, 3 = severe, and $4=$ very severe damage) as described previously $(13,18)$ : (a) airway lining epithelial loss, (b) deposition of extracellular matrix (ECM), (c) leukocyte infiltration, and (d) luminal obliteration due to granulation tissue formation and/or fibrosis. 

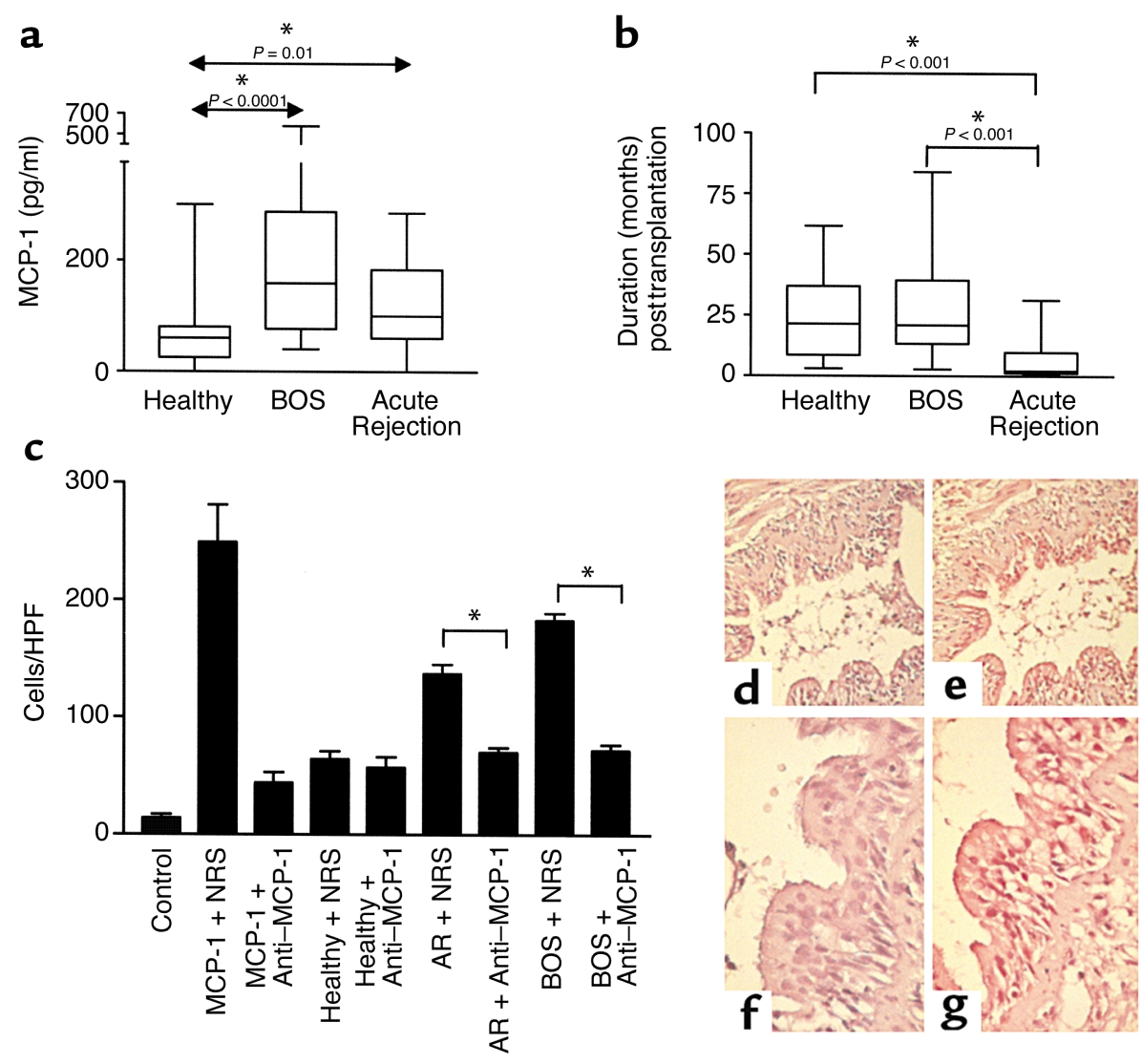

\section{Figure 1}

(a) MCP-1 protein levels in unconcentrated BALF from healthy lung transplant recipients compared with those of lung transplant recipients with BOS and lung transplant recipients with acute lung allograft rejection, displayed using a box plot summary. Horizontal line represents the median, the box encompasses the 25th to 75th percentiles, and the error bars encompass the 10th to 90th percentiles for MCP-1 protein levels. (b) The period, in months after lung transplantation, when the BALF was obtained for the three groups displayed, using a box plot summary. (c) BALF MCP-1 from patients with acute rejection and BOS is biologically active as determined by mononuclear cell chemotaxis. There was significantly more chemoattraction to the acute rejection and BOS BALF compared with that of healthy transplant recipients. Neutralizing antibodies to MCP-1 inhibited chemoattraction in the acute rejection and BOS groups, but not the healthy group. ${ }^{*} P<0.05$. ( $\left.\mathbf{d}-\mathbf{g}\right)$ BOS lung section $(\times 400)$. ( $\mathbf{d}$ and $\mathbf{f}$ ) Lack of nonspecific staining with NRS; (e and $\left.\mathbf{g}\right)$ anti-human MCP- 1 antibodies demonstrating immunolocalization to columnar epithelium and mononuclear cells. HPF, high power field; AR, acute rejection.

MCP-1 and MIP-2 protein ELISA. MCP-1 and MIP-2 protein was quantitated using a modification of a double-ligand method as described previously $(11,12)$.

FACS analysis of infiltrating lenkocyte populations. Tracheal single-cell suspension preparations were made from harvested tracheas using a method described previously (12). Single-cell suspensions $\left(5 \times 10^{6}\right.$ cells $\left./ \mathrm{ml}\right)$ were stained with Tricolor conjugated anti-murine CD45 (Caltag Laboratories Inc., South San Francisco, California, USA) followed by PE-conjugated mouse antimurine CD3e, CD4, CD8a, FITC anti-murine Ly-6G (PharMingen, San Diego, California, USA), FITC-conjugated anti-murine MOMA-2 (Seratec Inc., Raleigh, North Carolina, USA), or isotype controls. The cell suspensions were analyzed as described previously $(11,12)$.

Total RNA isolation and RT-PCR amplification. Total cellular RNA from tracheal tissue was isolated as described previously (9-12). Total RNA was determined, and $1 \mu \mathrm{g}$ of total RNA was reversed transcribed into cDNA and amplified using Access RT-PCR kit (Promega Corp.,
Madison, Wisconsin, USA) and specific primers for murine MCP-1, CCR2, procollagen type I and III, and our housekeeping gene, $\beta$-actin. The primers used were as follows: $5^{\prime}$-TTG-TAA-CCA-ACT-GGG-ACG-ATA-TGG-3' (sense) and $5^{\prime}$-GAT-CTT-GAT-CTT-CAT-GGT-GCT-AGG-3' (anti-sense) for $\beta$ actin (764 bp); $5^{\prime}$-AAG-CGT-GAC-AGAGAC-CTG-CAT-AGT-GGT-GG-3' (sense) and 5'-CCA-CCACTA-TGC-AGG-TCT-CTG-TCA-CGC-TT-3' (anti-sense) for murine MCP-1 (320 bp); 5'-CAC-GAA-GTA-TCC-AAG-AGCTT- $3^{\prime}$ (sense) and $5^{\prime}$-CAT-GCT-CTT-CAG-CTT-TTT-AC- $3^{\prime}$ (anti-sense) for murine CCR-2; $5^{\prime}$-TGG-TGC-CAA-GGGTCT-CAC-TGG-C-3' (sense) and 5'-GGA-CCT-TGT-ACACCA-CGT-TCA-CC-3' (anti-sense) for procollagen type I (352 bp); and 5'-GCA-GTC-CAA-CGT-AGA-TGA-ATT-GG-3' (sense) and 5'-GAA-GGC-CTG-GTG-GAC-CAG-CTG-G-3' (anti-sense) for procollagen type III (251 bp). After amplification, the sample was separated on an agarose gel containing ethidium bromide and bands visualized and photographed using an ultraviolet transilluminator (19). The image densities were measured with NIH 
a

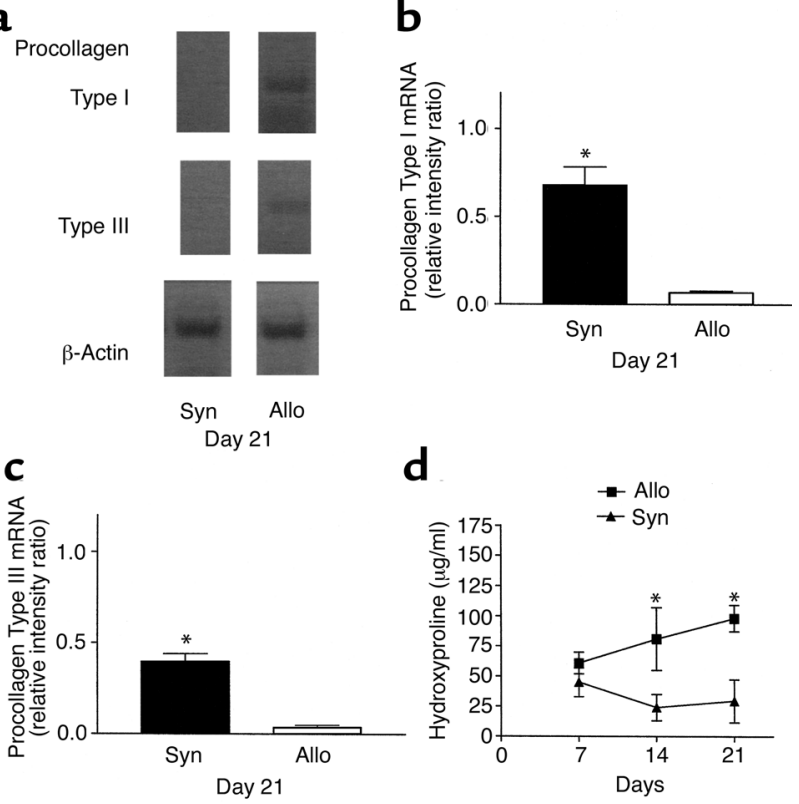

Figure 2

Procollagens type I and III and hydroxyproline levels are increased in allografts undergoing BOS. (a) RT-PCR determination of procollagen type I and III mRNA from allografts and syngeneic controls, compared with $\beta$-actin at day 21 . (b and $\mathbf{c}$ ) Semiquantitative results are expressed as a ratio of each PCR product to $\beta$-actin band density ( $n=4$ groups, in which each group represents four pooled tracheas at each time point). ${ }^{*} P<0.05$. (d) Hydroxyproline measurements from allografts and syngeneic controls at days 7,14 , and $21(n=4$ groups, in which each group represents two pooled tracheas at each time point). ${ }^{*} P<0.05$.

Image software, version 1.62 (NIH, Bethesda, Maryland, USA), and semiquantitative results were expressed as a ratio of each RT-PCR product to $\beta$-actin density.

Hydroxyproline assay. Total tracheal collagen was determined by analysis of hydroxyproline as described previously (11).

Statistical analysis. Data were analyzed on a Dell PC (Dell Computer Corp., Austin, Texas, USA) using the Statview 4.5 statistical package (Abacus Concepts Inc., Berkeley, California, USA). Comparisons were evaluated by the unpaired $t$ test. Nonparametric data were evaluated by Mann-Whitney analyses for statistical significance and displayed using a box plot summary. The plot's horizontal line represents the median; the box encompasses the 25th to 75th percentiles; and the error bars encompass the 10th to 90th percentiles. Continuous data were expressed as mean \pm SEM. Data were considered statistically significant if $P$ values were 0.05 or less.

\section{Results}

Elevated levels of biologically active $M C P-1$ are associated with both human acute lung allograft rejection and human BOS. We first determined whether MCP-1 was present in the lungs from patients undergoing BOS. MCP-1 levels in BALF were significantly elevated in patients with BOS, compared with the healthy recipients $(210 \pm 36$ vs. $72 \pm$ 13 , respectively) $(P<0.0001)$ (Figure 1a). There were no significant differences in duration after transplantation between these groups $(28.2 \pm 4.8$ vs. $24.8 \pm 3.2$ months, respectively) $(P>0.05)$, although there were differences between the acute rejection and healthy groups as expected ( $6.2 \pm 1.8$ vs. $24.8 \pm 3.2$ months, respectively) $(P<0.001)$ (Figure 1b). Given that acute rejection is the greatest risk factor for the development of $\mathrm{BOS}$, we evaluated the role of MCP-1 in the continuum of lung allograft rejection from acute to chronic rejection and determined MCP-1 levels in BALF from patients with acute rejection. Similar to the findings in the BOS group, we found elevated levels of MCP-1 in the acute rejection group compared with the healthy group (118 \pm 16 vs. $72 \pm 13$; respectively) $(P=0.01)$ (Figure 1a).

The elevated levels of MCP-1 in the BALF from both the acute rejection and BOS groups were biologically active as assessed by mononuclear cell chemotaxis (Figure 1c). Neutralizing antibodies to MCP-1 significantly attenuated the chemoattractant activity in both the acute rejection and BOS groups compared with NRS (Figure 1c). There was no significant effect found with the neutralizing antibodies to MCP-1 or NRS in the healthy group (Figure 1c). Immunohistochemical staining was used to localize antigenic MCP-1 in lung biopsies from patients with BOS. Predominant cellular sources of MCP-1 were columnar epithelial cells and the mononuclear cells (Figure 1, d-g).

Murine BOS involves increased expression of procollagen type I and III, deposition of ECM, airway obliteration, and epithelial injury. To determine MCP-1's contribution to the pathogenesis of BOS, we used a murine model system of BOS
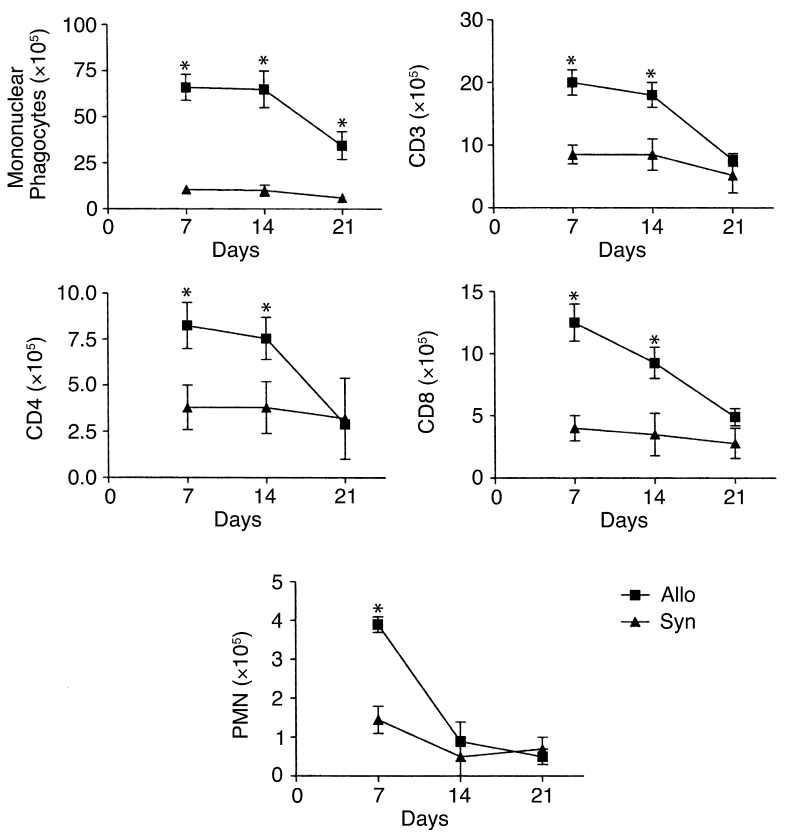

Figure 3

FACS analysis of leukocyte cell surface markers CD3, CD4, CD8, Ly-6G (PMN), and MOMA-2 (mononuclear phagocytes) from tracheal allografts undergoing BOS compared with syngeneic controls ( $n=4$ groups, in which each group represents four pooled tracheas at each time point). ${ }^{*} P<0.05$. 
a

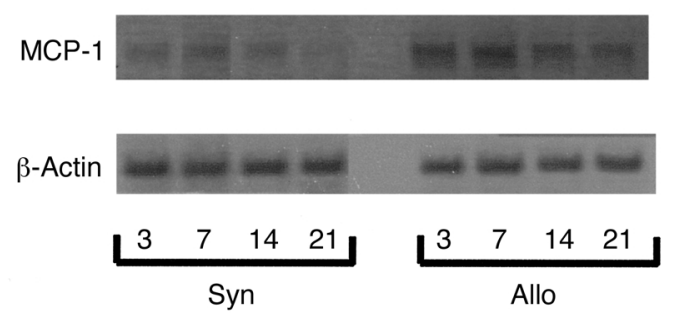

C

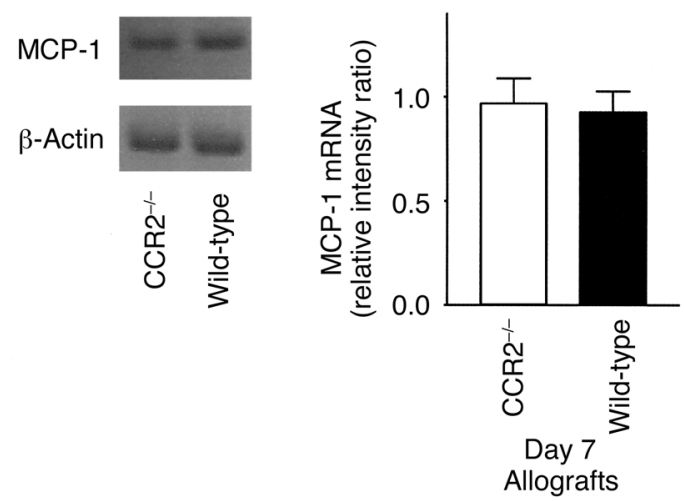

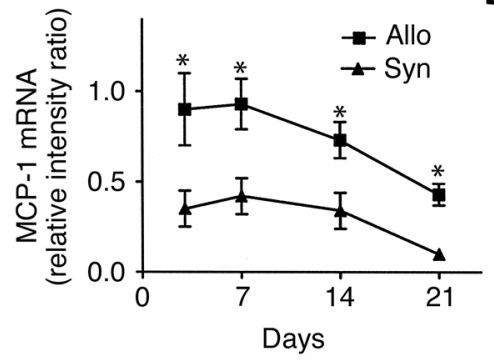

b
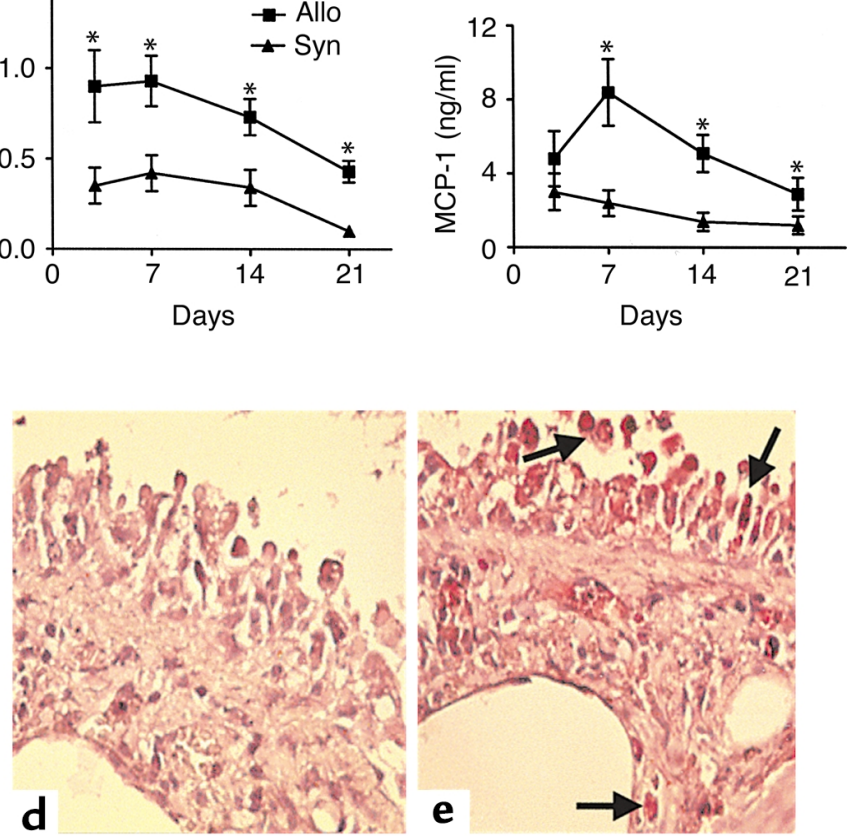

Figure 4

MCP-1 mRNA and protein levels are markedly elevated in murine allografts undergoing BOS. (a) RT-PCR determination of MCP-1 mRNA from allografts and syngeneic controls, compared with $\beta$-actin at days $3,7,14$, and 21 . Semiquantitative results are expressed as a ratio of each PCR product to $\beta$-actin band density ( $n=4$ groups, in which each group represents four pooled tracheas at each time point). ${ }^{*} P<0.05$. (b) ELISA measurements of MCP-1 protein levels from allografts and syngeneic controls at days 3-21 ( $n=4$ groups, in which each group represents four pooled tracheas at each time point). ${ }^{*} P<0.05$. (c) MCP-1 mRNA in allografts from CCR2 ${ }^{--}$(BALB/c tracheas to CCR2 $2^{--}$) versus CCR2 $2^{+/+}\left(B A L B / c\right.$ tracheas to CCR2 $\left.2^{++}\right)$mice at day 7. (d and e) Day 7 murine BOS section $(\times 400)$. (d) Lack of nonspecific staining with NRS; (e) anti-murine MCP-1 antibodies demonstrating immunolocalization to injured airway columnar epithelium and mononuclear cells.

$(13-15,18)$. Because BOS is a fibroproliferative disease, we determined whether there was expression of procollagen types (I and III) in murine allografts at day 21, a time point of maximal fibro-obliteration. Procollagens types (I and III) mRNA expression was markedly upregulated in the allografts compared with syngeneic controls (Figure 2 , a-c). We confirmed these findings, demonstrating a temporal increase in total collagen (hydroxyproline) levels in the allografts compared with syngeneic controls (Figure 2d). In addition, morphometric analysis of $\mathrm{H} \& \mathrm{E}$ and Masson's Trichrome staining of histopathological sections of allografts demonstrated significant increases in ECM deposition, airway obliteration, and loss of epithelial integrity compared with those found in syngeneic controls on days 7-21 (data not shown).

There is increased leukocyte infiltration during murine BOS. Our human data suggest that MCP-1 expression is a pivotal event in the continuum from acute to chronic of lung allograft rejection, through the persistent recruitment of peribronchial mononuclear cells. The magnitude and characteristics of leukocyte infiltration into allografts during murine BOS were quantitated by FACS (Becton Dickinson and Co., Franklin Lakes, New Jersey, USA) analysis. The most striking finding was the extensive number of mononuclear phagocytes infiltrating the allograft that was persistent during days 7-21, compared with that of syngeneic controls (Figure 3, top left). The numbers of infiltrating mononuclear phagocytes were markedly greater than the numbers of lymphocytes (CD3, CD4, and CD8) and neutrophils throughout the 21-day time course (Figure 3). This was confirmed by morphometric analysis of allograft histopathological sections demonstrating an aggressive transmural leukocyte infiltration peaking at day 7 , persisting at day 14 , and giving way to chronic inflammatory/fibroproliferative changes at day 21 (data not shown). In contrast, syngeneic controls demonstrated minimal peritracheal infiltration of leukocytes (data not shown).

$M C P-1 m R N A$ and protein are elevated during murine BOS. Demonstrating a mononuclear phagocyte dominant leukocyte infiltration into the allograft with subsequent development of fibro-obliteration lead us to determine the full kinetics of MCP-1 during the pathogenesis of BOS. MCP-1 mRNA from allografts peaked at day 7 and remained significantly elevated throughout the 21-day time course, compared with that of syngeneic controls (Figure 4a). A similar pattern was seen with MCP-1 protein (Figure 4b).

Immunohistochemical staining was used to localize antigenic MCP-1 in allografts at day 7. We found MCP-1 staining localized predominantly to airway columnar epithelium and mononuclear cells (Figure 4, $\mathrm{d}$ and e). In contrast there was virtually undetectable localization of MCP-1 in syngeneic controls at day 7 (data not shown). 
a
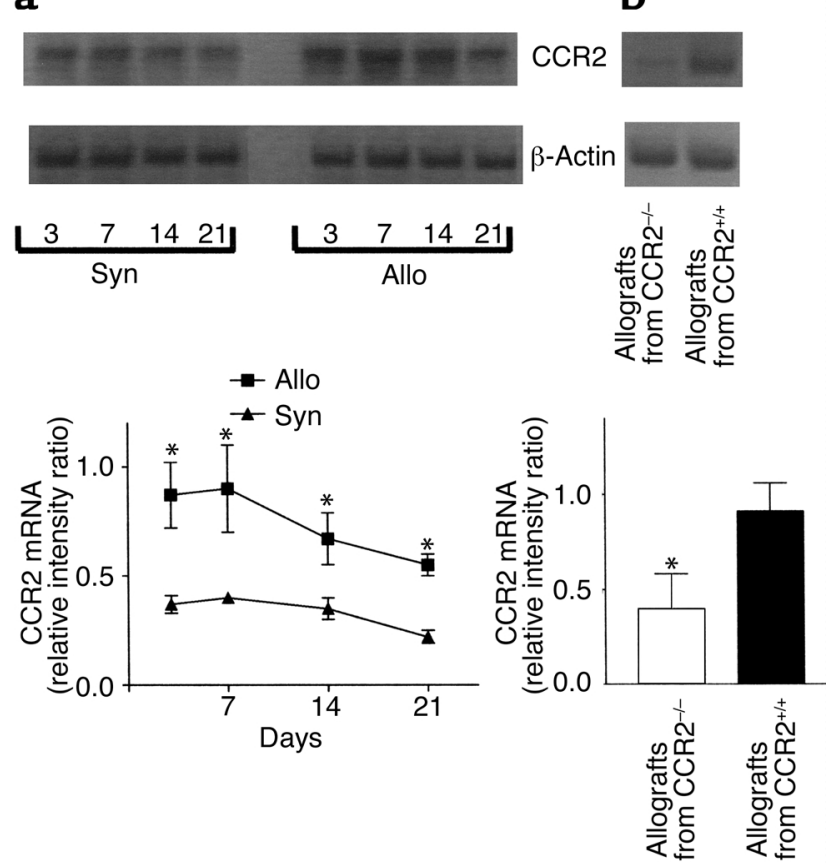

Day 7

\section{Figure 5}

CCR2 mRNA expression is markedly elevated in murine allografts undergoing BOS. (a) RT-PCR determination of CCR2 mRNA from allografts and syngeneic controls, compared with $\beta$-actin at days 3 , 7, 14, and 21. (b) CCR2 mRNA in allografts from CCR2-/- (BALB/c tracheas to $\left.\mathrm{CCR} 2^{-/-}\right)$versus $\mathrm{CCR} 2^{+/+}\left(\mathrm{BALB} / \mathrm{c}\right.$ tracheas to $\mathrm{CCR} 2^{+/+}$) mice at day 7 . Semiquantitative results are expressed as a ratio of each PCR product $/ \beta$-actin band density $(n=4$ groups, in which each group represents four pooled tracheas at each time point). ${ }^{*} P<0.05$.

CCR2 expression parallels both MCP-1 expression and recruitment of mononuclear cells during murine BOS. CCR2 is the major mononuclear cell receptor for the ligand, MCP-1. CCR2 mRNA from allografts was upregulated at day 3 , peaked at day 7 , and remained significantly elevated through day 21, compared with that of syngeneic controls (Figure 5a). CCR2 expression paralleled both MCP-1 expression and mononuclear cell recruitment (Figure 3 and Figure 4, $a$ and $b$ ).

$\mathrm{CCR} 2^{-/-}$mice display defective recruitment of mononuclear phagocytes during murine BOS. With MCP-1 correlating with the recruitment of mononuclear cells and expression of CCR2, we determined whether inhibiting MCP-1/CCR2 signaling could attenuate leukocyte recruitment. We used a genetic strategy using CCR2 $2^{-/}$versus $\mathrm{CCR} 2^{+/+}$mice as the recipients of $\mathrm{BALB} / \mathrm{c}$ tracheal allografts. We found reductions in infiltrating mononuclear phagocytes from the CCR2 $-/-$ mice through day 21 by FACS analysis (Figure 6a). Importantly, this occurred without significant reductions in lymphocytes (CD3, CD4, and CD8) (Figure 6, b-d). Furthermore, neutrophils were reduced in the CCR2 ${ }^{-/-}$recipients at day 7 (Figure 6 e). These results were further confirmed by semiquantitative analysis of histopathological sections demon- strating significant reductions in infiltrating leukocytes from CCR2 $2^{-/-}$recipients (Figure 7 ).

The increased production of potent leukocyte chemoattractants, such as MCP-1 and MIP-2, are characteristic of allograft rejection (20). We found no significant difference between MCP-1 protein levels in allografts from $\mathrm{CCR} 2^{-/-}$versus $\mathrm{CCR} 2^{+/+}$mice throughout the 21-day time course (Figure 6f). However, MIP-2 levels were reduced at day 7 in allografts from $\mathrm{CCR} 2^{-/-}$mice, which correlated with the reduction of neutrophils in the CCR2-/- mice at day 7 (Figure $6 \mathrm{~g}$ ).

CCR2 $-1-$ mice demonstrated a protective effect on ECM deposition, airway obliteration, and epithelial injury. With significant reductions of recruited leukocytes into the allografts from CCR2 $2^{-/}$mice, we evaluated whether this affected matrix deposition, airway obliteration, and epithelial integrity. Specifically, decreased recruitment of activated leukocytes to the allograft basement membrane and submucosa may delay the fibro-obliterative changes of BOS. Histopathological analysis of allografts from the CCR2--- recipients on days 3-14 demonstrated minimal ECM deposition, airway obliteration, and loss of epithelial cell integrity with occasional areas of hypertrophy and hyperplasia (Figure 7). Day 21 allografts from the CCR2-/- recipients demonstrated an increase in ECM matrix deposition, moderate airway obliteration, and a moderately severe epithelial injury including areas of metaplasia (Figure 7). Day 3 allografts from the $\mathrm{CCR} 2^{+/+}$mice demonstrated injury consistent with ischemia-reperfusion injury (data not shown). Day 7-14 allografts demonstrated a temporal increase from moderate to severe ECM deposition, airway obliteration, and severe epithelial injury with increased metaplasia (Figure 7). Day 21 allografts from the $\mathrm{CCR} 2^{+/+}$recipients demonstrated very severe ECM deposition, airway obliteration, and a denuded, metaplastic, thin layer of remnant epithelium, all consistent with progressive fibroproliferative changes (Figure 7). We further quantitated these findings by measuring hydroxyproline levels. There was a significant reduction in hydroxyproline levels at days 14 and 21 from CCR2-mice compared with those from CCR $2^{+/+}$mice (Figure $8 a)$. To investigate whether this reduction in allograft inflammation and fibro-obliteration was due to a difference in immune responses between the CCR2 $2^{-/}$and $\mathrm{CCR} 2^{+/+}$mice, we studied MCP- 1 and CCR 2 expression in allografts from the CCR2 $2^{-/-}$(BALB/c tracheas transplanted to CCR2 $\left.2^{-/}\right)$versus CCR2 ${ }^{+/+}(\mathrm{BALB} / \mathrm{c}$ tracheas transplanted to $\mathrm{CCR} 2^{+/+}$) recipient mice. There was no difference in MCP-1 expression (Figure 4c). There was less expression of CCR2 from the CCR2 $2^{-/}$versus $\mathrm{CCR} 2^{+/+}$allografts do to donor BALB/c cells (Figure $5 \mathrm{~b})$. In addition, we performed experiments: (CCR2 $2^{--}$ on $\mathrm{C} 57 \mathrm{BL} / 6$ background tracheas to $\mathrm{BALB} / \mathrm{c}$ ) versus $\left(\mathrm{CCR} 2^{+/+}\right.$on $\mathrm{C} 57 \mathrm{BL} / 6$ background tracheas to $\mathrm{BALB} / \mathrm{c}$ ) mice and found no difference in hydroxyproline levels at day 21 (Figure 8b). Moreover, to further probe the role of MCP-1/CCR2 signaling during the pathogenesis of BOS, we performed experiments using neutraliz- 
a

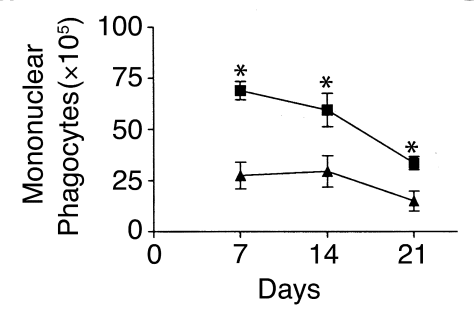

b

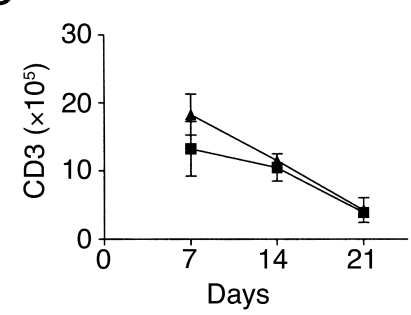

C

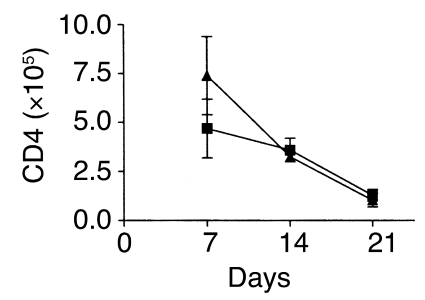

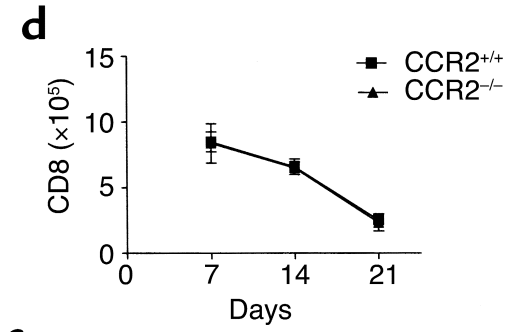

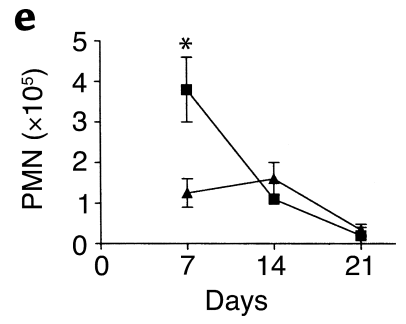

f
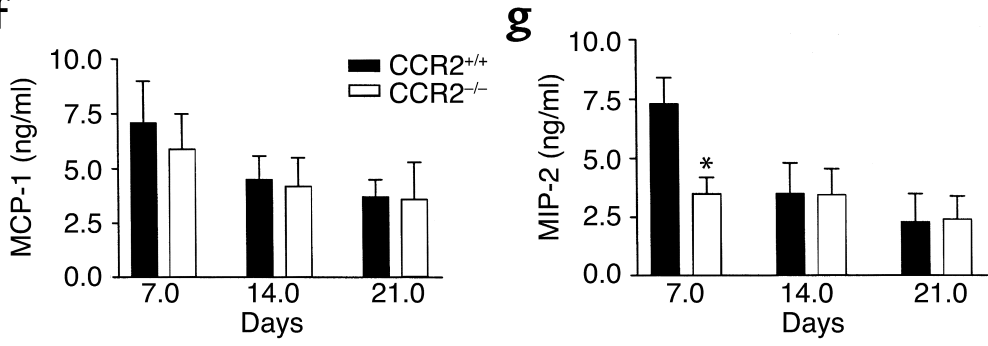

\section{Figure 6}

(a-e) FACS analysis of leukocyte cell surface markers CD3, CD4, CD8, Ly-6G (PMN), and MOMA-2 (mononuclear phagocytes) in tracheal allografts from CCR2 $2^{-/-}$versus CCR2 $2^{+/+}$recipient mice over our 21 -day time course $(n=4$ groups, in which each group represents four pooled tracheas at each time point). ${ }^{*} P<0.05$. ( $f$ and $\mathbf{g}$ ) ELISA measurements of protein level in allografts from CCR2 ${ }^{-/-}$versus CCR2 ${ }^{+/+}$mice at days 7, 14, and 21. (f) MCP-1 and (g) MIP-2 ( $n=4$ groups, in which each group represents four pooled tracheas at each time point). ${ }^{*} P<0.05$.

ing antibodies to MCP-1 in our model (BALB/c tracheas to C57BL/6 mice). Neutralization of endogenous MCP-1 significantly reduced hydroxyproline levels at day 21 compared with NRS (Figure 8c).

\section{Discussion}

BOS results from a chronic immunological/inflammatory insult to the allograft airways. This leads to a persistent peribronchiolar leukocyte infiltration, followed by an aberrant repair and fibro-obliteration of the airways. In this study, we hypothesized that the persistent expression of MCP-1 during the allogeneic response, with subsequent recruitment of activated mononuclear cells expressing CCR2, is pivotal for the promotion of the pathogenesis of BOS.

We assessed whether MCP-1 was associated with the continuum of lung allograft rejection from human acute to chronic rejection. We found significant increases in levels of MCP-1 in BALF from patients with acute rejection and BOS, compared with those found in healthy lung transplant recipients. The elevated levels of MCP-1 were biologically active as determined by mononuclear cell chemotaxis. Immunolocalization demonstrated that both airway epithelium and mononuclear cells were the predominant cellular sources of MCP-1. This association between MCP-1 and both acute and chronic allograft rejection has been identified in animal models of both heart and renal transplantation $(21,22)$. Both acute and chronic lung allograft rejection depend on the recruitment of peribronchial leukocytes, which, when persistent, eventually leads to fibro-obliteration of the allograft airway (14). This suggests that MCP-1 produced predominantly from recruited and trapped mononuclear cells is critically important in the continuum from acute to chronic lung allograft rejection by causing persistent accumulation of peribronchiolar leukocytes. In further support of these findings, MCP-1 has been associated with other human chronic inflammatory/fibroproliferative diseases such as idiopathic pulmonary fibrosis and abdominal adhesions $(23,24)$. Together these findings suggest an association between augmented levels of MCP-1 and the fibrogenesis involved in human chronic inflammatory/fibroproliferative diseases.

To determine whether MCP-1/CCR2 signaling contributes to the pathogenesis of human BOS, we performed translational studies using a murine model of BOS. We found allografts undergoing BOS had increased procollagen expression, hydroxyproline levels, and histopathology demonstrating increased matrix deposition. Consistent with our human data, we found marked elevations of MCP-1 in allografts undergoing BOS compared with those found in syngeneic controls. Immunolocalization demonstrated 


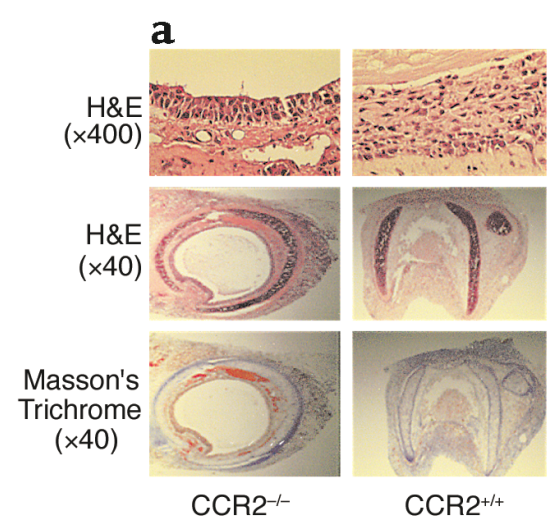

Day 14 b

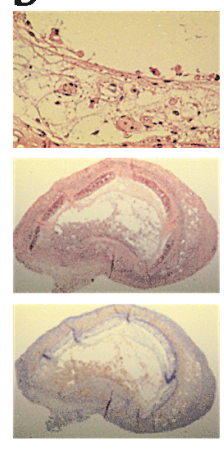

$\mathrm{CCR}^{-1-}$

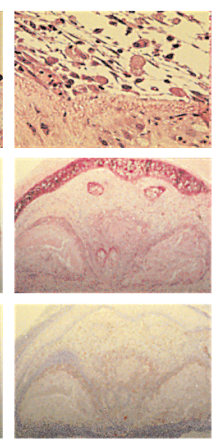

$\mathrm{CCR}^{+1+}$
Day 21

\section{Figure 7}

Representative photomicrographs $(\times 400$ and $\times 40)$ of the histopathology of tracheal allografts from CCR2-/- versus $\mathrm{CCR} 2^{+/+}$recipient mice. (a) Day 14 allografts from $\mathrm{CCR} 2^{+/+}$recipient mice demonstrate marked transmural leukocyte infiltration, moderate ECM deposition, and severe airway obliteration epithelial injury. In contrast, allografts from $\mathrm{CCR} 2^{-/-}$recipient mice show marked reductions in leukocyte infiltration, matrix deposition, epithelial injury, and airway obliteration. (b) Day 21 allografts from $\mathrm{CCR}^{+/+}$recipient mice demonstrate persistent transmural leukocyte infiltration, very severe ECM deposition, airway obliteration, and epithelial injury. In contrast, allografts from $\mathrm{CCR} 2^{-/-}$recipient mice show marked reductions in leukocyte infiltration, matrix deposition, airway obliteration, and epithelial injury. airway columnar epithelium and mononuclear cells as the predominant sources of MCP-1. Furthermore, MCP-1 levels during murine BOS paralleled the recruitment of mononuclear cells. The association between MCP-1 and a mononuclear phagocyte-predominant leukocyte infiltration is supported by other studies of solid organ chronic allograft rejection $(14,21)$. In rat models of chronic cardiac and lung allograft rejection, there were increases in expression of MCP-1 that remained markedly elevated throughout their respective time courses $(14,21)$. Furthermore, in a rat model of chronic renal allograft rejection, increased MCP-1 expression correlated best with the recruitment of macrophages compared with that of lymphocytes (22). These findings demonstrate the important relationship between augmented expression of MCP-1 and mononuclear phagocyte infiltration during chronic allograft rejection.

With regard to the prolonged production of MCP-1 in the continuum from acute to chronic allograft rejection, previous studies have demonstrated that the proximal proinflammatory cytokine TNF- $\alpha$ is expressed during an allogeneic response in human mononuclear cell mixed lymphocyte response (MLR) (25). In addition, TNF- $\alpha$ induced the prolonged expression of MCP-1 during this response (25), and in vitro neutralization of TNF- $\alpha$ downregulated the production of MCP-1 (25). We have confirmed these data with murine splenocytes MLR (C57BL/6 responder cells to BALB/c stimulator cells), and murine coculture experiments between murine C57BL/6 PBMCs and BALB/c fibroblasts (data not shown). Further- more, during murine BOS there is a marked elevation of TNF- $\alpha$ in allografts compared with that of syngeneic controls (data not shown).

CCR2 expression paralleled both MCP-1 and mononuclear cell recruitment during BOS. Similarly, other studies of chronic inflammatory diseases such as murine experimental autoimmune encephalomyelitis and rat induced glomerular fibrosis have demonstrated similar findings $(26,27)$. These studies support our data, suggesting an association between augmented levels of MCP-1 and recruitment of mononuclear cells expressing CCR2 during chronic inflammation/fibrogenesis.

To determine the role of MCP-1/CCR2 signaling in leukocyte recruitment during the pathogenesis of murine BOS, we performed allogeneic tracheal transplants in $\mathrm{CCR} 2^{-/-}$versus $\mathrm{CCR} 2^{+/+}$recipient mice. We found allografts from CCR2 $2^{-/}$mice demonstrated significant reductions in mononuclear phagocytes
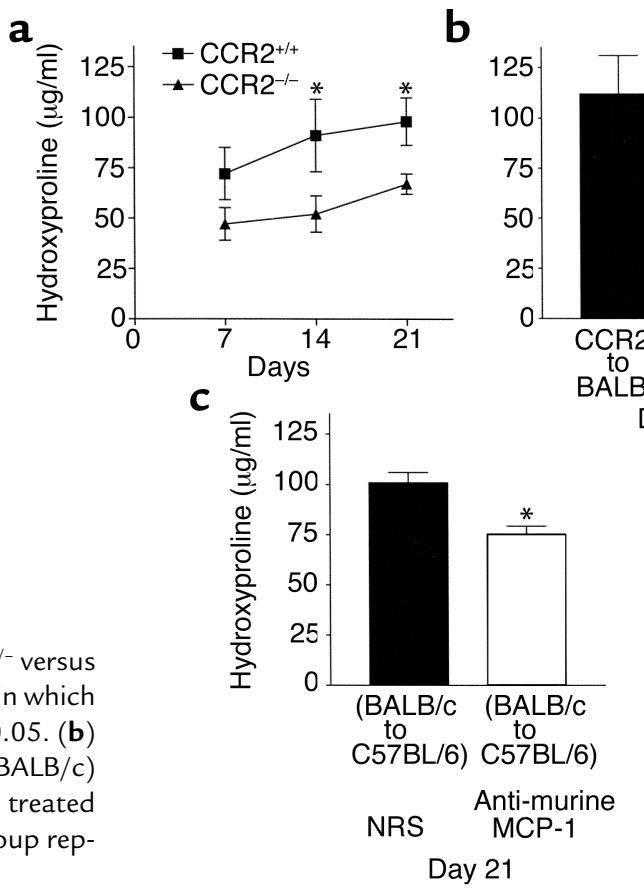
throughout the entire 21-day time course. Importantly, this was not accompanied by significant reductions in lymphocytes. This demonstrates that the phenotypically distinct mononuclear phagocyte expressing CCR2 is pivotal during the pathogenesis of BOS. Other studies using CCR2 $-/$ mice have shown that these mice have impaired mononuclear cell adhesion to endothelium, specifically a defect in mononuclear phagocyte extravasation and accumulation in tissue $(17,28)$. Furthermore, the importance of mononuclear phagocytes during rejection was demonstrated in a human study of chronic renal allograft rejection. There was a significant increase in infiltrating interstitial macrophages without any change in interstitial lymphocytes (29). Similarly, in another human study there was a correlation between deterioration in renal graft function and infiltrating mononuclear phagocytes (30). In addition, macrophage depletion in rat models of both orthotopic corneal and small bowel transplantation significantly attenuated rejection $(31,32)$. These studies, together with our data demonstrating reductions of mononuclear phagocyte-derived chemokines, suggest the decreased number of recruited mononuclear phagocytes interacting with $\mathrm{T}$ cells and other stromal cells leads to the reduction of specific cytokines, chemokines, and growth factors, in part, attenuating BOS.

With regard to neutrophils, there was an early reduction at day 7 , possibly reflecting a protective role for the CCR2 $-/-$ mutation during the neutrophil predominant leukocyte extravasation of ischemia reperfusion. In vitro, MCP-1 is chemotactic for mononuclear cells, but not neutrophils (33). However, neutrophils in an inflammatory milieu can express CCR2, potentially enabling their chemotaxis to MCP-1 (34). In addition, day 7 CCR $2^{--}$allografts had reduced levels of the neutrophil chemoattractant MIP-2, as compared to $\mathrm{CCR} 2^{+/+}$allografts. This is similar to what was demonstrated in a murine model of Cryptococcus pneumonia, where in vivo depletion of MCP-1 resulted in reduced numbers of monocytes as well as their secreted cytokines and chemokines, thereby indirectly decreasing neutrophil infiltration (9).

Finding significant reductions of infiltrating leukocytes into the allografts from CCR2 $2^{--}$recipient mice, we next assessed these effects on fibro-obliterative changes. Histopathological assessment of allografts from the $\mathrm{CCR} 2^{-/-}$mice demonstrated significantly less matrix deposition, airway obliteration, and epithelial injury throughout the 21-day time course. These findings are similar to the effect of granuloma formation in CCR2-mice, which demonstrate a decrease in recruitment of mononuclear phagocytes associated with decreased fibroproliferation and expression of procollagens (16, 17, 28). Similarly, in a murine model of atherosclerosis, CCR $2^{-/-}$crossed with ApoE ${ }^{-/-}$mice displayed a marked reduction in vessel obliteration secondary to a reduction in recruited mononuclear phagocytes within the lesions $(35,36)$. Furthermore, in a murine model of influenza $\mathrm{A}$-induced pneumonitis, $\mathrm{CCR} 2^{-/}$mice demonstrated a marked reduction in pulmonary inflammatory cells and airway epithelial injury (37). Similarly, we demonstrated a significant reduction in early epithelial injury, implying decreased recruitment of mononuclear phagocytes and their secreted chemokines, and that decreased infiltration of neutrophils to the submucosal regions of the airways has a protective role during early alloreactive epithelial airway injury. However, the increased injury at day 21 suggests that the inhibition of MCP-1/CCR2 signaling only attenuates and delays airway epithelial injury. Furthermore, allograft bronchiolar epithelium can express MHC class II antigens and has antigen-presenting functions similar to those of dendritic cells (38). Like the dendritic cell, the epithelial cells may have paradoxical interactions with mononuclear phagocytes depending on the local milieu (39). This suggests epithelialmononuclear phagocyte interaction during the chronic inflammatory phase of BOS even in the CCR2 $2^{-/-}$mice may have an injurious effect on epithelial cells.

To determine whether a significant difference in the immune response from the CCR2 $2^{-/}$versus CCR2 $2^{+/+}$ mice could account for the attenuation of BOS, we evaluated levels of MCP-1 from BALB/c tracheal allografts from $\mathrm{CCR} 2^{-/-}$versus $\mathrm{CCR} 2^{+/+}$recipient mice and found no difference. Experiments using CCR2 ${ }^{-/-}$versus $\mathrm{CCR} 2^{+/+}(\mathrm{C} 57 \mathrm{BL} / 6$ background) tracheas transplanted into recipient $\mathrm{BALB} / \mathrm{c}$ mice demonstrated no difference in fibro-obliteration. Similarly, there was no difference in MCP-1 and CCR2 expression from these allografts (data not shown). Therefore, it is the unique nature of the CCR $2^{-/-}$recipient mice that dictates the reduction of recruited mononuclear phagocytes in response to the production of MCP-1 during allogeneic injury. Furthermore, neutralizing antibodies to MCP-1 in allografts $(\mathrm{BALB} / \mathrm{c}$ tracheas transplanted into recipient C57BL/6 mice) significantly attenuated fibro-obliteration, solidifying the involvement of MCP-1/CCR2 signaling in BOS. Taken together, these findings suggest a direct role for MCP-1/CCR2 signaling in the recruitment of mononuclear phagocytes, granulation tissue formation, and lumenal obliteration in response to an injured airway from an allogeneic response.

In conclusion, we have demonstrated that MCP-1, as well as its interaction with its major mononuclear cell receptor CCR2, plays a pivotal role in mediating the persistent peribronchiolar mononuclear cell infiltration that leads to fibro-obliteration during BOS. The fact that there are still mononuclear phagocytes in our allografts from CCR $2^{-/-}$recipients, albeit reduced in number, suggests that other chemokines may have a role during BOS. The study of MCP-1/CCR2 signaling and future studies of other CC and CXC chemokines and there interaction with their appropriately expressed receptors will lead to the development of new paradigms to understand the pathogenesis of chronic lung allograft rejection. The findings in this study may ultimately result in novel therapies designed to attenuate MCP-1/CCR2 signaling and lead to better interventions in the treatment of BOS. 


\section{Acknowledgments}

This work was supported, in part, by grants from the NIH (HL-04493 to J.A. Belperio; HL-68694 and HL03906 to M.P. Keane; P50HL-67665 to M.P. Keane and R.M. Strieter; and CA87879, P50CA90388, and HL66027 to R.M. Strieter). John A. Belperio is a recipient of the ALA Research Grant Award, RG-019-B, and the Stein Oppenheimer Award 2001.

1. Arcasoy, S.M., and Kotloff, R.M. 1999. Lung transplantation. N. Engl. J. Med. 340:1081-1091.

2. Trulock, E.P. 1997. Lung transplantation. Am. J. Respir. Crit. Care Med. 155:789-818.

3. Carr, M.W., Roth, S.J., Luther, E., Rose, S.S., and Springer, T.A. 1994 Monocyte chemoattractant protein 1 acts as a T-lymphocyte chemoattractant. Proc. Natl. Acad. Sci. USA. 91:3652-3656.

4. Taub, D.D., et al. 1995. Monocyte chemotactic protein-1 (MCP-1), -2, and -3 are chemotactic for human $\mathrm{T}$ lymphocytes. J. Clin. Invest. 95:1370-1376

5. Gong, J.H., Ratkay, L.G., Waterfield, J.D., and Clark-Lewis, I. 1997. An antagonist of monocyte chemoattractant protein 1 (MCP-1) inhibits arthritis in the MRL-lpr mouse model. J. Exp. Med. 186:131-137.

6. Charo, I.F., et al. 1994. Molecular cloning and functional expression of two monocyte chemoattractant protein 1 receptors reveals alternative splicing of the carboxyl-terminal tails. Proc. Natl. Acad. Sci. USA. 91:2752-2756

7. Yousem, S.A., et al. 1996. Revision of the 1990 working formulation for the classification of pulmonary allograft rejection: Lung Rejection Study Group. J. Heart Lung Transplant. 15:1-15.

8. DiGiovine, B., et al. 1996. Bronchoalveolar lavage neutrophilia is associated with obliterative bronchiolitis after lung transplantation: role of IL8. J. Immunol. 157:4194-4202.

9. Huffnagle, G.B., et al. 1995. The role of monocyte chemotactic protein-1 (MCP-1) in the recruitment of monocytes and CD4+ T cells during a pulmonary Cryptococcus neoformans infection. J. Immunol. 155:4790-4797.

10. Huffnagle, G.B., et al. 1997. Macrophage inflammatory protein-1alpha (MIP-1alpha) is required for the efferent phase of pulmonary cell-mediated immunity to a Cryptococcus neoformans infection. J. Immunol. 159:318-327.

11. Keane, M.P., et al. 1999. Neutralization of the CXC chemokine, macrophage inflammatory protein-2, attenuates bleomycin-induced pulmonary fibrosis. J. Immunol. 162:5511-5518.

12. Belperio, J.A., et al. 2000. The role of the CC chemokine, RANTES, in acute lung allograft rejection. J. Immunol. 165:461-472.

13. Boehler, A., et al. 1998. Adenovirus-mediated interleukin-10 gene transfer inhibits post-transplant fibrous airway obliteration in an animal model of bronchiolitis obliterans. Hum. Gene Ther. 9:541-551.

14. Boehler, A., et al. 1999. Upregulation of T-helper 1 cytokines and chemokine expression in post-transplant airway obliteration. Am.J. Respir. Crit. Care Med. 159:1910-1917.

15. Hertz, M.I., Jessurun, J., King, M.B., Savik, S.K., and Murray, J.J. 1993. Reproduction of the obliterative bronchiolitis lesion after heterotopic transplantation of mouse airways. Am. J. Pathol. 142:1945-1951.

16. Warmington, K.S., et al. 1999. Effect of C-C chemokine receptor 2 (CCR2) knockout on type-2 (schistosomal antigen-elicited) pulmonary granuloma formation: analysis of cellular recruitment and cytokine responses. Am. J. Pathol. 154:1407-1416.

17. Boring, L., et al. 1997. Impaired monocyte migration and reduced type 1 (Th1) cytokine responses in C-C chemokine receptor 2 knockout mice. J. Clin. Invest. 100:2552-2561.
18. Boehler, A., et al. 1997. Lymphocytic airway infiltration as a precursor to fibrous obliteration in a rat model of bronchiolitis obliterans. Transplantation. 64:311-317.

19. Chumakov, K.M. 1994. Reverse transcriptase can inhibit PCR and stimulate primer-dimer formation. PCR Methods Appl. 4:62-64.

20. Kondo, T., Novick, A.C., Toma, H., and Fairchild, R.L. 1996. Induction of chemokine gene expression during allogeneic skin graft rejection. Transplantation. 61:1750-1757.

21. Russell, M.E., et al. 1995. Upregulation of cytokines associated with macrophage activation in the Lewis-to-F344 rat transplantation model of chronic cardiac rejection. Transplantation. 59:572-578.

22. Nadeau, K.C., Azuma, H., and Tilney, N.L. 1995. Sequential cytokine dynamics in chronic rejection of rat renal allografts: roles for cytokines RANTES and MCP-1. Proc. Natl. Acad. Sci. USA. 92:8729-8733.

23. Zeyneloglu, H.B., et al. 1998. The role of monocyte chemotactic protein1 in intraperitoneal adhesion formation. Hum. Reprod. 13:1194-1199.

24. Antoniades, H.N., et al. 1992. Expression of monocyte chemoattractant protein 1 mRNA in human idiopathic pulmonary fibrosis. Proc. Natl. Acad. Sci. USA. 89:5371-5375.

25. Christensen, P.J., et al. 1993. Characterization of the production of monocyte chemoattractant protein-1 and IL- 8 in an allogeneic immune response. J. Immunol. 151:1205-1213.

26. Fife, B.T., Huffnagle, G.B., Kuziel, W.A., and Karpus, W.J. 2000. CC chemokine receptor 2 is critical for induction of experimental autoimmune encephalomyelitis. J. Exp. Med. 192:899-906.

27. Schadde, E., et al. 2000. Expression of chemokines and their receptors in nephrotoxic serum nephritis. Nephrol. Dial. Transplant. 15:1046-1053.

28. Kuziel, W.A., et al. 1997. Severe reduction in leukocyte adhesion and monocyte extravasation in mice deficient in CC chemokine receptor 2. Proc. Natl. Acad. Sci. USA. 94:12053-12058.

29. Yanagisawa, T., et al. 1997. Relationship between macrophage infiltration of renal allografts and chronic renal impairment. Transplant. Proc. 29:2783-2786.

30. Ramos, E.L., et al. 1995. Thromboxane synthase expression in renal transplant patients with rejection. Transplantation. 59:490-494.

31. Slegers, T.P., et al. 2000. Effect of macrophage depletion on immune effector mechanisms during corneal allograft rejection in rats. Invest. Ophthalmol. Vis. Sci. 41:2239-2247.

32. Fryer, J., et al. 1996. Influence of macrophage depletion on bacterial translocation and rejection in small bowel transplantation. Transplantation. 62:553-559.

33. Loetscher, P., Dewald, B., Baggiolini, M., and Seitz, M. 1994. Monocyte chemoattractant protein 1 and interleukin 8 production by rheumatoid synoviocytes. Effects of anti-rheumatic drugs. Cytokine. 6:162-170.

34. Johnston, B., et al. 1999. Chronic inflammation upregulates chemokine receptors and induces neutrophil migration to monocyte chemoattractant protein-1. J. Clin. Invest. 103:1269-1276.

35. Dawson, T.C., Kuziel, W.A., Osahar, T.A., and Maeda, N. 1999. Absence of CC chemokine receptor-2 reduces atherosclerosis in apolipoprotein E-deficient mice. Atherosclerosis. 143:205-211.

36. Boring, L., Gosling, J., Cleary, M., and Charo, I.F. 1998. Decreased lesion formation in CCR2-/- mice reveals a role for chemokines in the initiation of atherosclerosis. Nature. 394:894-897.

37. Dawson, T.C., Beck, M.A., Kuziel, W.A., Henderson, F., and Maeda, N. 2000. Contrasting effects of CCR5 and CCR2 deficiency in the pulmonary inflammatory response to influenza A virus. Am. J. Pathol. 156:1951-1959.

38. Yousem, S.A., et al. 1990. HLA-class II antigen expression in human heart-lung allografts. Transplantation. 49:991-995.

39. Gong, J.L., McCarthy, K.M., Rogers, R.A., and Schneeberger, E.E. 1994. Interstitial lung macrophages interact with dendritic cells to present antigenic peptides derived from particulate antigens to T cells. Immunology. 81:343-351. 